Marketingkonzepte für das EG-Öko-Audit-System

\title{
Gezielte Strategien notwendig
}

\begin{abstract}
Die EG-Öko-Audit-Verordnung (EMAS) ist binnen fünf Jahren von vielen Unternehmen der Mitgliedsstauten angenommen worden. Allerdings fiel die Teilnahme in einzelnen Ländern sehr unterschiedlich aus, und es ist strittig, ob die Entwicklung als „erstaunlich rasant" oder "eher verhalten" zu bezeichnen ist. Eine weitere Verbreitung des Systems erscheint auf jeden Fall notwendig. Konzepte und Strategien des Marketing können hier wertvolle Hilfestellungen liefern.
\end{abstract}

Von Werner Warmbier

G rundsätzlich sind drei Strategien denkbar: das System wird für Bereiche geöffnet, die bisher keinen Zugang hatten (,Markterweiterung"), bereits teilnehmende Unternehmen werden zur weiteren Teilnahme veranlaßt (,Kundenbindung"), noch nicht teilnehmende Unternehmen werden von der Teilnahme am System überzeugt (,Neukundengewinnung“).

Ein erster Schritt in Richtung Markterweiterung ist in Deutschland mit der Erweiterungsverordnung des Umweltauditgesetzes (UAG) vom 3. Februar 1998 erfolgt. Der aktuelle Diskussionsstand in Brüssel deutet darauf hin, daß demnächst für die EU eine totale Marktöffnung erfolgt, so daß Unternehmen und Institutionen aus allen relevanten Sektoren am System teilnehmen können (1).

Im Rahmen der Kundenbindung gilt es, Unternehmen, die für einen Validierungszyklus Teilnehmer sind, auch darüber hinaus ,,bei der Stange zu halten“. Beim geltenden Prinzip der freiwilligen Beteiligung können die Teilnehmer im Grunde jederzeit das System wieder verlassen. Diese Gefahr wird jedoch allgemein als gering erachtet.

\section{Wollen Sie einen Überblick über die bisherigen Inhalte des Informationsdienstes "Ökologisches Wirtschaften ${ }^{\prime ?}$ ?}

\author{
Dann wählen Sie sich einfach \\ ein unter
}

\section{http://www.oekom.de}

unter dieser Adresse können

Sie zugleich auch bestellen.
Firmen und sonstige Organisationen überlegen sich ihren Entschluß zu einer ersten Teilnahme recht genau. Sie wollen den einmal getätigten Aufwand später nicht ohne weiteres abschreiben und fürchten das Risiko eines Imageverlustes, der aus einem Austritt resultieren könnte.

Kritisch wird es nur, wenn es EMAS auf Dauer nicht gelingt, sich als umweltpolitisches Instrument mit anerkannt hohem Stellenwert zu etablieren. Dann wird nicht nur das Risiko des Imageverlustes bestehen, vielmehr werden Teilnehmer möglicherweise sogar als Verkäufer umweltpolitischer Mogelpackungen betrachtet und deshalb aus dem System flüchten. Um ein hohes Niveau zu erreichen und langfristig zu halten, wäre eine Art Qualitätssicherung für das System sinnvoll.

Formal sind für die Teilnehmer mit dem Validierungszyklus und für die Gutachter mit dem UAG und seinen Verordnungen Qualitäts-,,Kontrollen “ bereits angelegt. EMAS gibt bekanntlich keine Mindestwerte oder Standards vor, die bei der Verbesserung der Umweltauswirkungen zu erreichen sind. Damit wird es für den Betrachter von außen schwierig, Fortschritte zu beurteilen oder Vergleiche zwischen Teilnehmern anzustellen. Um dies zu erleichtern und die Transparenz des Systems zu erhöhen, eignet sich beispielsweise das Konzept des Benchmarking, das Vergleichsdaten liefert und auf die Optimierung von Verbesserungsmaßnahmen zielt (2).

\section{Wie neue Kunden gewinnen?}

Ansatzpunkte zur Gewinnung neuer Kunden werden im Marketing häufig anhand des AIDAModells (Attention - Interest - Desire - Action) entwickelt. Dieses Modell unterstellt, daß die Entscheidung der Kunden in Phasen erfolgt, in denen jeweils speziell zugeschnittene Informationen bereitzustellen sind. Für die Verbreitung von EMAS waren die Aktivitäten, die auf die frühe Pha- se des Erweckens von Aufmerksamkeit zielen, bisher allgemein gehalten, ohne allzu sehr auf spezielle Informationsbedürfnisse einzelner Zielgruppen einzugehen. Dies hat mit dazu beigetragen, daß trotz einer positiven Entwicklung der Teilnahme der Bekanntheitsgrad von EMAS insgesamt noch eingeschränkt ist. Nach einer Studie des ISO-Instituts zur Erforschung sozialer Chancen kennen zwei Drittel der kleinen und mittleren Unternehmen EMAS noch gar nicht (3).

Es stellt sich die Frage, ob es sinnvoll ist, angesichts der großen Zahl potentieller Teilnehmer mit ganz unterschiedlichen Ansprüchen und Reaktionsweisen breit angelegte, allgemein gehaltene Informationskampagnen durchzuführen. Solche Aktionen sind teuer und ihr Wirkungsgrad ist in der Regel begrenzt. Wenn Unternehmen also nicht undifferenziert angesprochen werden sollen, wo sind dann gezielt Ansatzpunkte effizienterer Maßnahmen zu suchen?

Die von EMAS ausgehenden, insgesamt zu erwartenden positiven Umweltwirkungen hängen u.a. vom Umfang der derzeitigen Umweltbelastungen $\mathrm{ab}$, die mit den jeweiligen Tätigkeiten potentiell teilnehmender Unternehmen verbunden sind. Um besonders positive Umwelteffekte $\mathrm{zu}$ erreichen, ließen sich daher diejenigen ,hot spots“ (seien es einzelne Unternehmen oder ganze Sektoren) identifizieren, bei denen eine Erweiterung der Teilnahme am System besonders umweltwirksam wäre. Hier könnte dann EMAS gezielt und konzentriert eingebracht werden, im Sinne des AIDAModells angefangen bei ersten Informationen zum System, bis hin zu angepaßten Hilfsmitteln und Fördermaßnahmen, die die Teilnahme erleichtern. Natürlich trägt unabhängig vom aktuellen relativen Grad seiner Umweltbelastung jeder weitere Teilnehmer zu einer Verbesserung der Situation bei, insofern geht es auch darum, eine möglichst große Zahl von Unternehmen für einen Beitritt zu gewinnen.

\section{- Übliche Strategien problematisch}

Hierzu lassen sich bezüglich des Ansatzpunktes und der Wirkungsrichtung der Maßnahmen allgemein „Push“- und „Pull“-Strategien unterscheiden. „Push“ besagt, daß man versucht, etwas in den Markt hinein zu drücken. So würde z.B. ein Hersteller seinen Einfluß beim Großhandel geltend machen, um den Absatz seiner Produkte zu fördern, dieser wiederum beim Einzelhandel, der dann selbst den Verbraucher bewirbt. Beim „Pull“ zieht der Verbraucher das Produkt in den Markt, indem 
er es beim Einzelhandel nachfragt, der wiederum beim Großhandel usw. Wenn der Hersteller in diesem Fall den Absatz seines Produktes fördern will, muß der Verbraucher es natürlich kennen und wollen.

Beim derzeitigen Stand der Dinge erscheinen Pull-Strategien kaum aussichtsreich. Das Produkt EMAS ist noch viel zu wenig bekannt. Es ist auch anders als manche Umweltlabel sehr erklärungsbedürftig, was Konzept, Ziele und Methodik anbelangt; wahrscheinlich wird es sich einem Teil der Verbraucher nie erschließen. Durch das Verbot der Produktwerbung bleibt weiterhin die Tatsache der Teilnahme dort verborgen, wo der Verbraucher seine eigentliche „Macht“ ausüben kann, nämlich beim Kauf.

Beim Prinzip der freiwilligen Teilnahme am System sind Push-Strategien ebenfalls nicht geeignet, in größerem Umfang Unternehmen in das System zu zwingen. Dies hätte allenfalls Aussicht auf Erfolg, wenn umfangreiche Sondervergünstigungen eingeräumt würden.

Auch für das Ziel, eine möglichst große Zahl potentieller Teilnehmer zu erreichen, sind derzeit also unspezifische Aktionen im Sinne breit gestreuter Aufklärungskampagnen wenig sinnvoll. Es sind Bereiche zu suchen, in denen die höchsten Ausbreitungseffekte zu vermuten sind.

\section{Maßnahmen konzentrieren}

Wesentlich ist in diesem Zusammenhang, daß EMAS systemimmanente Elemente besitzt, die über die Grenzen der Betriebe hinausgehen. Der Anhang der Verordnung hebt an mehreren Stellen darauf $a b$, daß die Teilnehmer auch in ihrem Umfeld auf die Verbesserung des Umweltverhaltens hinwirken müssen. Hiermit sind insbesondere Auftragnehmer, Vertragspartner, Lieferanten und Kunden der Teilnehmer angesprochen.

Es sind also solche „Epizentren“ zu identifizieren, die sich am besten eignen, in ihrem Umfeld andere Unternehmen zur Teilnahme zu bewegen, die dann ihrerseits den Impuls weitergeben. Hier lassen sich konzentrierte Aktionen ansetzen mit unterschiedlicher Zielrichtung hinsichtlich einer Tiefen- oder Breitenwirkung.

Eine Tiefenwirkung ergibt sich in den Wertschöpfungsketten von Industrie und Handel, die über zahlreiche Kunden-Lieferanten-Beziehungen miteinander verbunden sind. Ein Impuls ginge von der Möglichkeit aus, die Teilnahme unternehmensübergreifend zu attestieren, etwa: ,Alle Lieferanten dieses Standortes sind auditierte Betriebe“. Hierdurch angeregt würde ein besse- res Ineinandergreifen der Prozesse und eine stärkere Vernetzung der Wertschöpfungsketten und Marketingkanäle umweltaktiver Unternehmen sowie eine erhöhte Motivation zur Integration von Nicht-Teilnehmern.

Für die Breitenwirkung erscheinen insbesondere die Kommunen interessant. Zu ihren Lieferanten, Auftragnehmern und Vertragspartnern zählt ein bunter Mix von Firmen unterschiedlicher Branchen und Größenordnungen, häufig mit Regionalbezug. Nachdem ihnen die Möglichkeit zur Teilnahme eröffnet wurde, gilt es nicht nur, die Kommunen als Teilnehmer an strategisch wichtigen Kristallisationspunkten $\mathrm{zu}$ gewinnen, sondern sie auch $\mathrm{zu}$ befähigen und dabei $\mathrm{zu}$ unterstitzen, kleine und mittlere Unternehmen beim Beitritt zum System zu fördern. Darüiber hinaus haben die Kommunen ja auch eine gewisse Informationspllicht gegenüber den Bürgern und anderen Kunden, was die Breitenwirkung weiter erhöht.

Marketingstrategien für das Öko-Audit-System dürfen sich im übrigen nicht darin erschöpfen, Aktivitäten zur Werbung neuer Teilnehmer durchzufiuhren. Vielmehr sollten sie auch Aktionen für „alte“ Teilnehmer beinhalten, um mit ihnen Allianzen für die weitere Verbreitung des Systems zu bilden. Man kann dabei von einer weitgehend übereinstimmenden Interessenlage - wenn auch aus unterschiedlichen Motivationen heraus - zwischen externen Promotoren des Öko-AuditSystems und den Teilnehmern ausgehen. Erstere wollen EMAS bekannt machen, um den Teilnehmerkreis zu erweitern, letztere wollen über einen gesteigerten Bekanntheitsgrad des Systems die Bedeutung ihrer eigenen Teilnahme aufwerten.

\section{Anmerkungen}

(1) Vgl. auch die Diskussion in Ökologisches Wirtschaften, Heft 3-4/1998.

(2) Vgl. zum derzeitigen Diskussionsstand zu diesem Konzept z.B. Heinz Kottmann, Jens Clausen (Hg.): Ökologisches Benchmarking von Unternehmen. IÖW-Schriftenreihe 133/98.

(3) Ökologisches Wirtschaften 2/98, S. 3.

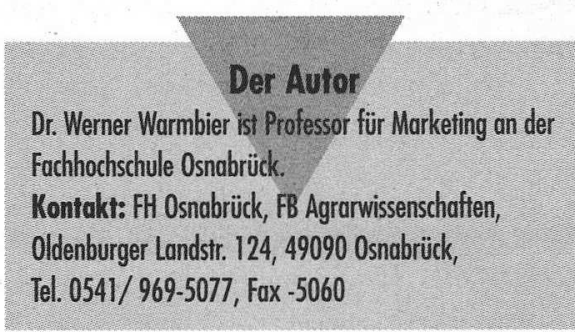

\section{Der Rat von Sachverständigen für Umweltfragen http://www.umweltrat.de \\ UMWELTSCHUTZ}

\section{Erreichtes sichern - Neue Wege gehen}

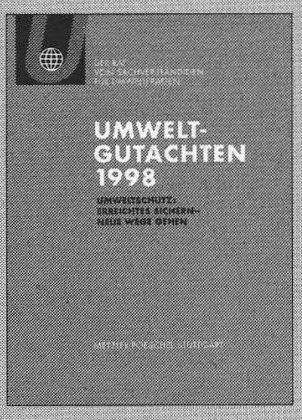

In seinem neuesten Umweltgutachten entwickelt der Umweltrat einen Verfahrensvorschlag zur Festlegung langfristiger Umweltqualităts- und Umwelthandlungsziele. In der generellen Politikbewertung werden Fragen zum Umweltgesetzbuch, zu den Selbstverpflichtungen der Wirtschaft und zur Öko-Audit-Verordnung behandelt. Weitere Schwerpunktthemen sind: Aspekte der Abfallwirtschaftspolitik, Umweltprobleme der Freisetzung gentechnisch veränderter Pflanzen, Umweltschutz und inter nationaler Handel sowie Umwelt, Freizeit und Tourismus.

Umweltgutachten 1998

Umweltschutz: Erreichtes sichern Neue Wege gehen

Best.-Nr. 7800206-97902

ISBN 3-8246-0561-9

390 Seiten, 9 Abb., 36 Tabellen.

Gebunden, DM 68,-

\section{Sondergutachten:}

Flächendeckend wirksamer Crundwasserschutz

Ein Schritt zur dauerhaft umweltgerechten Entwicklung

Best.-Nr. 7800114-97901

ISBN 3-8246-0560-0 208 Seiten, 37 Abb., 49 Tabellen. Kartoniert, DM 38,-

Paket Umweltgutachten 1998 und Sondergutachten

DM 88,-; Best.-Nr. 7800402-97907

Bestellung per Fax $(07071) 935393$ oder per eMail: staba@s-f-g.com Bitte geben Sie Ihre vollständige Lieferadresse an!

\section{METZLER POESCHEL-}


(c) 20I0 Authors; licensee IÖW and oekom verlag. This is an article distributed under the terms of the Creative Commons Attribution Non-Commercial No Derivates License (http://creativecommons.org/licenses/by-nc-nd/3.o/), which permits unrestricted use, distribution, and reproduction in any medium, provided the original work is properly cited. 